\title{
Xanthine Oxidase Activity in Type 2 Diabetes Mellitus Patients with and without Diabetic Peripheral Neuropathy
}

\author{
Dijana J. Miric, ${ }^{1}$ Bojana M. Kisic, ${ }^{1}$ Snezana Filipovic-Danic, ${ }^{2}$ Rade Grbic, ${ }^{3}$ Ilija Dragojevic, \\ Marko B. Miric, ${ }^{2}$ and Dragana Puhalo-Sladoje ${ }^{4}$ \\ ${ }^{1}$ Institute of Biochemistry, Medical Faculty, University of Pristina, Kosovska Mitrovica, Serbia \\ ${ }^{2}$ Clinics for Neurology and Psychiatry, Medical Faculty, University of Pristina, Kosovska Mitrovica, Serbia \\ ${ }^{3}$ Clinics for General and Orthopedics Surgery, Medical Faculty, University of Pristina, Kosovska Mitrovica, Serbia \\ ${ }^{4}$ Biochemical Laboratory, Medical Faculty, University of East Sarajevo, Foca, Bosnia And Herzegovina
}

Correspondence should be addressed to Dijana J. Miric; miric.dijana@gmail.com

Received 8 September 2016; Revised 22 October 2016; Accepted 26 October 2016

Academic Editor: Norman Cameron

Copyright (C) 2016 Dijana J. Miric et al. This is an open access article distributed under the Creative Commons Attribution License, which permits unrestricted use, distribution, and reproduction in any medium, provided the original work is properly cited.

\begin{abstract}
This study investigated the relationship between serum xanthine oxidase (XOD) activity and the occurrence of diabetic peripheral neuropathy (DPN) in type 2 diabetes mellitus (T2DM) patients. Serum XOD activity, ischemia-modified albumin (IMA), uric acid (UA), albumin, glycated hemoglobin (HbAlc), advanced glycation end products (AGE), total free thiols, atherogenic index of plasma (AIP), and body mass index (BMI) were measured in 80 T2DM patients (29 with and 51 without DPN), and 30 nondiabetic control subjects. Duration of diabetes, hypertension, medication, and microalbuminuria was recorded. Serum XOD activities in controls, non-DPN, and DPN were $5.7 \pm 2.4 \mathrm{U} / \mathrm{L}, 20.3 \pm 8.6 \mathrm{U} / \mathrm{L}$, and $27.5 \pm 10.6 \mathrm{U} / \mathrm{L}(p<0.01)$, respectively. XOD activity was directly correlated to IMA, UA, BMI, HbAlc, and AGE, while inversely correlated to serum total free thiols. A multivariable logistic regression model, which included duration of diabetes, hypertension, AIP, HbAlc, UA, and XOD activity, revealed HbAlc [OR = $1.03(1.00-1.05) ; p=0.034$ ] and XOD activity [OR $=1.07(1.00-1.14) ; p=0.036$ ] as independent predictors of DPN. Serum XOD activity was well correlated to several other risk factors. These results indicate the role of XOD in the development of DPN among T2DM patients.
\end{abstract}

\section{Introduction}

Diabetic peripheral neuropathy (DPN) is a late-stage microvascular complication that develops in nearly $50 \%$ patients during the course of type 2 diabetes mellitus (T2DM), affecting particularly low extremities. DPN is characterized by irreversible nerve structural and functional changes due to demyelination, axonal atrophy, and diminished regenerative potential, clinically presenting as a symmetric chronic pain, paraesthesia, and sensory loss. The development of DPN often worsens the quality of life [1], and increases the risk of cardiovascular morbidity, foot ulcerations, amputations, and overall mortality [2].

Although exact mechanisms are not fully understood, DPN is generally regarded as the consequence of chronic hyperglycemia-induced endothelial dysfunction, resulting in impaired endoneurial blood flow, ischemia, and nerve hypoxia [3]. Metabolic-vascular interactions in DPN are however highly complex and include diverse molecular pathways, like activation of protein kinase $\mathrm{C}$ and polyol metabolism, glycation and glycoxidation, low-grade inflammation, and excessive formation of reactive oxygen species (ROS), such as superoxide and hydrogen peroxide [4]. In vascular compartment these ROS can react with intrinsic vasodilator nitric oxide, and while the fall of nitric oxide levels would cause vasoconstriction, the resulting peroxynitrite could trigger the downstream events leading to nerve damage [5].

Because normal oxygen supply is fundamental for all tissues, impaired vasodilation is often associated with cellular energy crisis and increased breakdown of purine nucleotide to uric acid (UA) via xanthine oxidoreductase. Under physiological conditions this enzyme functions as dehydrogenase, 
but during hypoxia or after limited proteolysis it is converted to oxidase form (XOD). Unlike dehydrogenase, XOD more readily generates ROS able to impair vascular relaxation. In diabetic patients, hyperglycemia-induced endothelial dysfunction is chronic and not limited solely to the blood vessels irrigating nerve tissue but to variable extent occurs throughout the body. Moreover, XOD has been previously linked to oxidative damage in diabetes $[6,7]$ and diabetic cataract [8], as well as metabolic syndrome and its cardiovascular complications [9], and the role of XOD was also proposed in an experimental model of diabetic neuropathy [10].

As a molecular fingerprint of imminent hypoxia, serum UA was reported higher in T2DM than in nondiabetic subjects, especially in those with DPN [11]. During hypoxia, circulating serum albumin can undergo conformational changes at N-terminus aspartyl-alanyl-hystidyl-lysine sequence, which decrease its natural ability to bind cobalt and several other transition metal ions. The resulting ischemiamodified albumin (IMA) was originally associated with hypoxic conditions during myocardial ischemia [12] but was thereafter reported also in T2DM [13] and some diabetic complications [14]. However, there are missing data regarding serum XOD activity or IMA in DPN. Currently, there is no effective therapy to cure DPN, and even with good glycemic control the chance to develop DPN is relatively high among T2DM patients. Given that XOD could be a significant source of ROS in vascular compartment causing endothelial dysfunction, and a possible therapeutic target, this study was aimed at investigating the relationship between XOD and $\mathrm{DPN}$ in T2DM patients.

\section{Materials and Methods}

2.1. Study Participants. This study enrolled 80 patients previously diagnosed with T2DM, both sexes, who attended our local Clinical Hospital Center on regular basis for diabetes control. Not included were patients with significant motor deficits, recent cardiovascular or cerebrovascular events, overt renal or hepatic diseases, and retinopathy and those with foot ulcerations, amputations, and recent inflammatory disease or with known autoimmune or malignant disease. Excluded were also patients treated with alpha-lipoic acid or allopurinol. The control group was consisted of 30 age- and sex-matched subjects without DM, recruited from medical stuff and their relatives, who met the same exclusion criteria. This study was conducted in accordance with the Declaration of Helsinki, after informed consent from patients was provided. The institutional review board of the Medical Faculty Pristina (Kosovska Mitrovica) has approved this study.

DPN was defined as symmetrical sensorimotor polyneuropathy, diagnosed using a simplified scoring system for bedside examination, the Diabetic Neuropathy Symptom (DNS) score [15], followed by Michigan Neuropathy Screening Instrument (MNSI) scoring system. All subjects with DNS score $>1$ were further assessed using the MNSI. The MNSI consists of two parts: the first part is a 15 -item "yes or no" questionnaire about history of sensory and motor dysfunction (pain, temperature sensitivity, tingling, numbness, cramps, muscle weakness, feet ulcers or cracks, and amputation). The second part of MNSI encompasses examination of foot skin appearance, inspection for foot ulcers, examination of ankle reflexes, vibration perception testing using a $128-\mathrm{Hz}$ tuning fork test at the great toe, and fine touch sensation testing using a $10 \mathrm{~g}$ Semmes-Weinstein monofilament applied on the plantar sites of each foot. DPN was defined as MNSI questionnaire $\geq 7$ or MNSI examination score $>2[16]$.

Hypertension was defined as having systolic blood pressure $\geq 140 \mathrm{mmHg}$ or diastolic blood pressure $\geq 90 \mathrm{mmHg}$ or being on antihypertensive medication. Microalbuminuria, as a marker of incipient kidney damage, was defined as urinary albumin-to-creatinine ratio of 30-300 mg/g [17]. Body mass index (BMI) was calculated as the ratio of body weight $(\mathrm{kg})$ and square of body height $\left(\mathrm{m}^{2}\right)$.

2.2. Biochemical Methods. Venous blood was taken after an overnight fasting into vacutainer tubes without or with anticoagulant (EDTA) to obtain serum, plasma, or whole blood samples. The first morning urine sample taken into sterile urine containers during two months in three nonconsecutive days was provided for determination of urinary albumin and creatinine.

Serum XOD activity was measured according to the method of Roussos [18], as described earlier [8]. XOD activity was calculated after correction for preexisting uric acid, using molar absorbance of uric acid at $\lambda=293 \mathrm{~nm}$, of $\varepsilon=1.26 \times$ $10^{4} \mathrm{~L} \times \mathrm{M}^{-1} \times \mathrm{cm}^{-1}$. One unit of XOD activity was defined as $1 \mu \mathrm{mol} / \mathrm{min}$ uric acid formed at $37^{\circ} \mathrm{C}$. Serum IMA was measured using a colorimetric method described by BarOr et al. [12]. Results were expressed as units per milliliter serum $(\mathrm{IU} / \mathrm{mL})$. Concentration of total serum thiols, as an indicator of protein oxidative damage, was measured using Ellman's reagent [19]. Concentration of serum AGE was determined spectrofluorometrically by the method of Kalousová et al. [20] and expressed as relative fluorescence units (RFU). Concentration of advanced oxidation protein products (AOPP), as marker of chronic oxidative albumin damage, was determined from lipid-depleted plasma samples to avoid interference with lipid status, by the method of Anderstam et al. [21]. The results were expressed as $\mu \mathrm{mol} / \mathrm{L}$ Chloramine-T equivalents. Serum XOD, AGE, and AOPP were assessed from aliquoted samples $(0.5 \mathrm{~mL})$ kept at $-80^{\circ} \mathrm{C}$.

Concentrations of serum and urinary creatinine, serum total proteins, albumin, triglycerides, total cholesterol, HDLcholesterol, UA, and blood glycated hemoglobin (HbAlc) were measured on Cobas Integra 400 biochemical analyzer, using standard protocols. Urinary albumin was determined using Tinaquant Albumin Gen.2 assay kit (Roche Diagnostics $\mathrm{GmbH}$, Mannheim, Germany). The average of urinary albumin excretion was calculated for each patient from results of three nonconsecutive urine samples. Concentration of LDL-cholesterol was calculated using Friedewald's formula. Atherogenic index of plasma (AIP), as a surrogate marker of atherogenic dyslipidemia, was calculated according to Dobiasova, as the logarithm of triglycerides to HDL-cholesterol concentration ratio [22]. 
TABLE 1: Basic demographical and clinical parameters in control subjects and type 2 diabetes mellitus patients with and without diabetic peripheral neuropathy.

\begin{tabular}{|c|c|c|c|}
\hline & \multirow{2}{*}{ Control subjects $(n=30)$} & \multicolumn{2}{|c|}{ Type 2 DM patients } \\
\hline & & Non-DPN $(n=51)$ & $\mathrm{DPN}(n=29)$ \\
\hline Age (years) & $60.3 \pm 8.7$ & $61.5 \pm 9.3$ & $62.9 \pm 7.5$ \\
\hline Gender (male/female; $n$ ) & $13 / 17$ & $20 / 31$ & $13 / 16$ \\
\hline BMI $\left(\mathrm{kg} / \mathrm{m}^{2}\right)$ & $25.3 \pm 4.1$ & $29.3 \pm 4.1^{*}$ & $28.4 \pm 4.5^{*}$ \\
\hline Diabetes duration (years) & NA & $5.83 \pm 2.56$ & $6.39 \pm 2.98$ \\
\hline Microalbuminuria (yes/no; $n$ ) & NA & $15 / 36$ & $10 / 19$ \\
\hline uACR (mg/g) & NA & $15.6(11.8-20.7)$ & $23.4(15.6-35.2)$ \\
\hline Hypertension (yes/no; $n$ ) & $13 / 17$ & $32 / 19$ & $18 / 11$ \\
\hline Current smokers (yes/no; $n$ ) & $11 / 19$ & $24 / 27$ & $9 / 20$ \\
\hline \multicolumn{4}{|l|}{ Antidiabetic medication } \\
\hline (i) Oral antidiabetic drugs $(n)$ & & 37 & 18 \\
\hline (ii) Oral antidiabetic drugs + insulin $(n)$ & NA & 10 & 8 \\
\hline (iii) Insulin $(n)$ & & 4 & 3 \\
\hline Fasting blood glucose (mmol/L) & $5.07 \pm 1.19$ & $7.34 \pm 2.51^{*}$ & $8.07 \pm 3.04^{*}$ \\
\hline Total cholesterol (mmol/L) & $5.86 \pm 1.05$ & $6.81 \pm 1.53^{*}$ & $6.64 \pm 1.76^{*}$ \\
\hline HDL-cholesterol (mmol/L) & $1.74 \pm 0.56$ & $1.47 \pm 0.41^{*}$ & $1.23 \pm 0.41^{* \ddagger}$ \\
\hline LDL-cholesterol (mmol/L) & $2.60 \pm 0.87$ & $3.88 \pm 1.32^{*}$ & $4.01 \pm 1.45^{*}$ \\
\hline Triglycerides (mmol/L) & $2.16 \pm 0.74$ & $2.85 \pm 1.04^{*}$ & $2.77 \pm 0.95^{*}$ \\
\hline AIP [log (triglycerides/HDL)] & $0.114 \pm 0.229$ & $0.270 \pm 0.229^{*}$ & $0.366 \pm 0.238^{*}$ \\
\hline Serum creatinine $(\mu \mathrm{mol} / \mathrm{L})$ & $76.3 \pm 10.4$ & $77.5 \pm 10.7$ & $77.0 \pm 11.9$ \\
\hline Total proteins $(\mathrm{g} / \mathrm{L})$ & $76.9 \pm 3.6$ & $75.3 \pm 3.1$ & $75.9 \pm 3.7$ \\
\hline
\end{tabular}

Data are presented as mean values \pm standard deviation, frequencies $(n)$, or geometric mean and $95 \%$ confidence interval of the mean (in parenthesis). Differences between groups were tested by one-way ANOVA, Student's independent samples $t$-test, or chi-square test. DPN: diabetic peripheral neuropathy; BMI: body mass index; NA: not applicable; uACR: urinary albumin to urinary creatinine ratio; AIP: atherogenic index of plasma.

${ }^{*} p<0.05$ versus control group; ${ }^{\ddagger} p<0.05$ versus non-DPN group.

2.3. Statistical Methods. Data analyses were performed using MedCalc Software package (Mariakerke, Belgium). Frequency distribution and homogeneity of variance were tested by Kolmogorov-Smirnov test. Data were presented as either arithmetic mean $\pm \mathrm{SD}$, frequencies $(f)$, or median and 95\% confidence interval of the median. Differences between groups were tested by ANOVA and Student's independent samples $t$-test, or chi-square test, where appropriate. Correlation analysis was accomplished by calculation of Spearman's coefficient. The relationship between DPN, XOD, and other risk factors was investigated by multivariable logistic regression analysis. Statistically significant finding was considered if $p<0.05$.

\section{Results}

Basic demographical, clinical, and biochemical findings in controls and T2DM patients are presented in Table 1. Of 80 T2DM patients enrolled in the study, 29 were with DPN; the remaining $(n=51)$ comprised the non-DPN group. There were no significant differences between control and groups with DM, in terms of age and sex distribution, smoking status, presence of hypertension, serum creatinine, and total protein levels (Table 1). Twenty-five T2DM patients were with microalbuminuria, having urinary albumin to creatinine ratio within the range of $30-300 \mathrm{mg} / \mathrm{g}$. Patients with T2DM had significantly higher BMI than control subjects and were rather overweight $(n=31)$ or obese $(n=26)$.

Concentrations of fasting blood glucose, total cholesterol, HDL-cholesterol, LDL-cholesterol, triglycerides, and AIP significantly differed from controls in both groups with T2DM (Table 1). In comparison to non-DPN group, concentration of HDL-cholesterol was significantly lower in DPN group.

In the overall sample the median XOD activity was 16.4 U/L (95\% CI 13.8-20.4). Serum XOD activity and IMA concentration were higher in T2DM patients than in control subjects and significantly differed between DPN and nonDPN groups (Table 2). Serum albumin and total thiols were lower in DM patients than in controls and differed between DPN and non-DPN groups. Compared to controls, HbAlc and serum AGE were increased in DM patients, being higher in DPN than in non-DPN group; the occurrence of DPN also affected serum UA concentrations. Serum AOPP was higher in T2DM patients than in controls, but the difference between diabetic groups was insignificant (Table 2).

In patients with T2DM, serum XOD activity was directly correlated to BMI, the presence of hypertension, and levels of HbAlc, AGE, IMA, and UA and inversely correlated to serum albumin and total thiol groups concentrations (Table 3). Correlations between XOD activity and age, duration of diabetes, AIP, AOPP, and microalbuminuria were not significant. 
TABLE 2: Serum XOD activity, uric acid, IMA, and other biomarkers of protein modifications in control subjects and type 2 diabetes mellitus patients with and without diabetic peripheral neuropathy.

\begin{tabular}{|c|c|c|c|}
\hline & \multirow{2}{*}{ Control subjects $(n=30)$} & \multicolumn{2}{|c|}{ Type 2 DM patients } \\
\hline & & Non-DPN $(n=51)$ & $\mathrm{DPN}(n=29)$ \\
\hline $\mathrm{XOD}(\mathrm{U} / \mathrm{L})$ & $5.7 \pm 2.4$ & $20.3 \pm 8.6^{*}$ & $27.5 \pm 10.6^{* \ddagger}$ \\
\hline IMA (IU/mL) & $28.5 \pm 8.7$ & $34.7 \pm 12.4^{*}$ & $40.3 \pm 11.8^{* \ddagger}$ \\
\hline Albumin (g/L) & $44.1 \pm 2.4$ & $43.4 \pm 2.2$ & $42.1 \pm 2.2^{* \ddagger}$ \\
\hline $\mathrm{HbAlc}(\mathrm{mmol} / \mathrm{mol})$ & $35.2 \pm 11.8$ & $59.2 \pm 22.7^{*}$ & $72.7 \pm 22.8^{* \ddagger}$ \\
\hline Uric acid $(\mu \mathrm{mol} / \mathrm{L})$ & $246 \pm 70$ & $268 \pm 72$ & $298 \pm 68^{*}$ \\
\hline Total thiols $(\mu \mathrm{mol} / \mathrm{L})$ & $541 \pm 94$ & $407 \pm 118^{*}$ & $294 \pm 86^{* \ddagger}$ \\
\hline AGE (RFU) & $6.41 \pm 1.19$ & $7.26 \pm 1.44^{*}$ & $8.45 \pm 2.17^{* \ddagger}$ \\
\hline AOPP $(\mu \mathrm{mol} / \mathrm{L}$ Chloramine-T) & $38.7 \pm 9.4$ & $63.7 \pm 23.7^{*}$ & $65.3 \pm 26.0^{*}$ \\
\hline
\end{tabular}

Data are presented as mean values \pm standard deviation. Differences between groups were tested by one-way ANOVA and post hoc Student's $t$-test. XOD: xanthine oxidase; IMA: ischemia-modified albumin; AGE: advanced glycation end products; AOPP: advanced protein oxidation products; RFU: relative fluorescence units.

${ }^{*} p<0.05$ versus control group; ${ }^{\ddagger} p<0.05$ versus non-DPN group.

TABLE 3: Relationships between serum XOD activity and various clinical and biochemical variables in type 2 diabetes mellitus.

\begin{tabular}{lcc}
\hline & \multicolumn{2}{c}{ XOD (U/L) } \\
& Spearman's rho & $p$ value \\
\hline Age (years) & 0.192 & 0.089 \\
Diabetes duration (years) & 0.120 & 0.287 \\
HbAlc (mmol/mol) & 0.244 & 0.030 \\
BMI $\left(\mathrm{kg} / \mathrm{m}^{2}\right)$ & 0.275 & 0.015 \\
AIP $[\log ($ triglycerides/HDL) & 0.165 & 0.144 \\
Uric acid ( $\mu$ mol/L) & 0.250 & 0.026 \\
Albumin $(\mathrm{g} / \mathrm{L})$ & -0.299 & 0.002 \\
Total thiol groups ( $\mu$ mol/L) & -0.267 & 0.018 \\
AGE (RFU) & 0.260 & 0.021 \\
AOPP $(\mu \mathrm{mol} / \mathrm{L}$ Chloramine-T) & 0.020 & 0.857 \\
IMA (IU/mL) & 0.227 & 0.044 \\
Hypertension (yes versus no) & 0.229 & 0.042 \\
Microalbuminuria (yes versus no) & 0.188 & 0.095 \\
\hline
\end{tabular}

Correlation analysis was accomplished by calculation of Spearman's nonparametric coefficient (rho) in 80 type 2 DM patients.

We further analyzed the association between several clinical and biochemical variables and the occurrence of DPN. Included were duration of diabetes, HbAlc, AIP, hypertension, serum XOD, and UA levels. The multivariable logistic regression analysis $(B=-5.014$; chi-square $=20.023$; $p=0.0027$ ) clearly revealed HbAlc and serum XOD activity as independent predictors of DPN, whereas the influence of diabetes duration was of the borderline significance (Table 4).

\section{Discussion}

The major finding of the present study was significant elevation of serum XOD activity in patients with T2DM and an independent association between XOD activity and the occurrence of DPN. Serum XOD activity was well correlated to the levels of IMA and some other biomarkers of increased ROS formation. Furthermore, XOD activity was directly correlated to several risk factors relevant for the development of DPN, including hypertension, higher BMI, and concentrations of $\mathrm{HbAlc}$ and UA, indicating the role of $\mathrm{XOD}$ in the development of DPN.

$\mathrm{XOD}$ is a rate-limiting enzyme of purine catabolism to UA, during which high quantities of ROS are produced. We observed that the formation of ROS was specifically increased in DPN group, documented as decreased serum total free thiols and increased levels of oxidatively damaged molecules, like IMA and AGE, accompanied by higher concentration of serum UA (Table 2). These findings are consistent with the concept that increased ROS formation can contribute to the development of DPN $[4,23]$.

Serum total free thiol groups essentially originate from a single 34 cystein residue of albumin, which is abundantly present blood protein. Acting as nonenzymatic antioxidant and reducing agent these free thiols are rapidly oxidized after exposure to ROS, forming mixed disulfides and related sulfur-containing acids. In one previous study the loss of total serum thiols was found to correspond to the severity of diabetic microvascular complications and the development of DPN [24], and our current results support that finding.

There was also a significant increase in serum IMA concentrations in T2DM, especially in DPN group, in our study. Several other studies reported elevated serum IMA levels in diabetes, correspondingly to the presence or severity of complications, including peripheral arterial disease [14]. We observed that IMA concentrations were positively correlated to XOD activity, indicating that oxidative damage of circulating biomolecules in DPN can be, at least partly, inflicted by XOD-derived ROS. Although exact chemical mechanisms of IMA formation are still a matter of debate, it is generally accepted that IMA is formed due to oxidative damage of serum albumin N-terminus [14], specifically in hypoxic conditions, such as those created by dysfunctional blood vessels. In accordance to that, IMA is now regarded as a biomarker of widespread endothelial damage in a variety of pathological states, including T2DM [13]. However, in the absence of other biomarkers of endothelial damage these results should be cautiously interpreted. Besides IMA, the 
TABLE 4: Multivariable logistic regression analysis for association with diabetic peripheral neuropathy.

\begin{tabular}{lcccc}
\hline Independent predictors & $\beta$ & Std. error & $p$ value & OR $(95 \%$ CI $)$ \\
\hline Diabetes duration (years) & 0.182 & 0.096 & 0.058 & $1.20(0.99-1.45)$ \\
AIP [log (triglycerides/HDL)] & 2.293 & 1.318 & 0.082 & $9.91(0.75-131.17)$ \\
HbAlc (mmol/mol) & 0.026 & 0.012 & 0.034 & $1.03(1.00-1.05)$ \\
Serum uric acid $(\mu \mathrm{mol} / \mathrm{L})$ & 0.006 & 0.004 & 0.165 & $0.99(0.98-1.00)$ \\
Hypertension (yes versus no) & 0.687 & 0.564 & 0.223 & $1.99(0.66-6.00)$ \\
XOD (U/L) & 0.067 & 0.032 & 0.036 & $1.07(1.00-1.14)$ \\
\hline
\end{tabular}

occurrence of DPN was associated with higher serum UA and lower albumin levels, which are known risk factors of DPN. Also, 31\% of patients included in the study were microalbuminuric; thus the coexistence of decreased UA elimination rate and increased urinary albumin excretion rate as confounding factors cannot be excluded.

Still, increased serum levels of IMA and UA have been regarded as biomarkers widespread endothelial dysfunction in a variety of pathological states, including T2DM [13, 25]. Tissues irrigated by dysfunctional vessels inevitably suffer hypoxic and even anoxic conditions, which promote the transition of xanthine dehydrogenase to XOD form. Within vascular compartment the enzyme is found mostly at XOD form, attached to endothelial cell surface via sulfated glycosaminoglycan-rich receptors, and as a free enzyme in the blood. Both bound and free XOD can generate ROS able to modify circulating blood and vascular wall constituents, such as albumin and endothelium-derived nitric oxide. The reaction of nitric oxide and superoxide anion radical diminishes nitric oxide levels and yields peroxynitrite, which can act as toxic mediator inducing nerve injury [5]. At the same time, diminished availability of nitric oxide would exacerbate endothelial dysfunction, since it is implicated in regulation of vascular tone, as well as in prevention of platelet adhesion and aggregation and leukocytes adhesion to the vascular wall. Moreover, augmented serum XOD activity has been already linked to increased ROS formation in metabolic syndrome and its cardiovascular complications, clinical and experimental diabetes, and diabetic ocular complications [69]. To the best of our knowledge this is the first report of XOD in T2DM with DPN.

The majority of vascular XOD is believed to be of hepatic origin, nonspecifically released into the blood, particularly under hyperglycemic conditions [6]. It was therefore not surprising that $\mathrm{HbAlc}$ levels were directly correlated to XOD activity in our and some previous studies $[6,7]$, thereby confirming that glycemic control plays critical role in modulating XOD presence/activity within vascular compartment. Besides HbAlc, serum XOD activity was also correlated to AGEs, which are chemically heterogeneous products of protein nonenzymatic glycation and subsequent oxidation reactions. AGEs not only are biomarkers of glycoxidative protein damage but also act as toxic mediators after binding to specific receptors, like multiligand RAGE or macrophage scavenger receptors. In peripheral nerves RAGE are primarily expressed in endothelial and Schwann cells. It is of note that AGE-RAGE interaction facilitated endoneurial vascular dysfunction in peripheral nerves, leading to microangiopathy [26].

Etiology of DPN is however highly complex, since diverse though interrelated metabolic lifestyles and genetic factors can be involved [6]. For example, a prolonged ischemia and obesity can be both present in patients with T2DM and associated with higher levels of serum UA [27]. In fact, one recent study demonstrated that, beyond purine metabolism, $\mathrm{XOD}$ also plays a role in differentiation of adipocytes acting as a regulator of the nuclear receptor peroxisome proliferatoractivated receptor $\gamma$ activity, which is the key factor controlling the induction and maintenance of adipogenesis [28]. In the present study, serum XOD activity was correlated to BMI as well as to UA levels (Table 3). One probable explanation could be a high prevalence of overweight (38.7\%) and obese (23.5\%) diabetics, and since XOD is abundantly expressed in adipocytes it might cause increased secretion of UA from adipose tissue, especially during ischemia [29]. These results are consistent with those of Feoli et al., who reported a similar relationship in metabolic syndrome [9].

It has been previously shown that ROS formed in chronic hyperglycemia may induce unmyelinated and myelinated nerve fibers loss and impairment of endoneurial blood flow, resulting in nerve conduction velocity abnormalities [23]. Histologically, DPN is characterized by accumulation of macrophages in perivascular lesions [30]. Upon activation, macrophages can produce high quantities of ROS via $\mathrm{XOD}$, thereby causing local endothelial dysfunction, further impairment of endoneurial blood flow, and myelin degeneration. Overexpression of XOD is actually necessary step for macrophages activation and regulation of proinflammatory mediators and chemokines secretion [31, 32]. Furthermore, ROS generated by local macrophages via XOD may induce nerve axonal loss and demyelination, as demonstrated in a murine model of neuroinflammation [33].

Because of high content of polyunsaturated fatty acids nervous tissue is exceptionally susceptible to ROS; thus tight control of prooxidant enzyme activity is of great importance. Previously, Inkster et al. reported that inhibition of XOD with allopurinol, a structural analogue of hypoxanthine with both hypouricemic and anti-inflammatory effects, was able to prevent the loss of motor and sensory conduction velocity in an animal model of diabetes [10]. Their study demonstrated that inhibition of XOD improved blood flow in sciatic and cervical nerve ganglion, thereby suggesting that upregulated XOD present in nerve microvasculature, including endothelium and perivascular space, can cause 
neurovascular dysfunction leading to diabetic neuropathy. Allopurinol also prevented cardiac ischemia and impaired relaxation in an experimental model of insulin resistance [34], normalized endothelial function in T2DM patients with mild hypertension [35], alleviated oxidative injury and improved cardiovascular functions in diabetics in several intervention studies [36], and acted antinociceptive against various noxious stimuli in mice [37]. On the other side, peripheral neuropathy can be a side effect of allopurinol treatment, and this drug failed to prevent the progression of DPN in type 1 diabetes mellitus patients with mild-tomoderate cardiovascular autonomic neuropathy [38].

Nonetheless, the current study has demonstrated that augmented XOD activity was present within vascular compartment in T2DM patients diagnosed with DPN. Moreover, serum XOD activity was closely correlated to risk factors relevant for the development of DPN, including poor glycemic control, obesity, and serum IMA and UA levels, which are considered as markers of generalized endothelial dysfunction in diabetics. Our results therefore indicate that upregulated XOD can be an additional source of ROS in vascular compartment, implicated in chronic oxidative injury and endothelial dysfunction in T2DM patients with poor glycemic control, contributing overtime to the development of DPN.

\section{Competing Interests}

The authors declare no competing financial or other interests regarding this manuscript.

\section{Authors' Contributions}

All authors equally contributed to this paper.

\section{Acknowledgments}

The authors are thankful to Dr. Nedeljka Dostic for her excellent technical assistance.

\section{References}

[1] Z. Vukojević, T. Pekmezović, A. Nikolić et al., "Correlation of clinical and neurophysiological findings with health related quality of life in patients with diabetic polyneuropathy," Vojnosanitetski Pregled, vol. 71, no. 9, pp. 833-838, 2014.

[2] Y. Mochizuki, H. Tanaka, K. Matsumoto et al., "Association of peripheral nerve conduction in diabetic neuropathy with subclinical left ventricular systolic dysfunction," Cardiovascular Diabetology, vol. 14, no. 1, article 47, 2015.

[3] S. Tesfaye, "Epidemiology and etiology of diabetic peripheral neuropathies," Advanced Studies in Medicine, vol. 4, no. 10 G, pp. S1014-S1021, 2004.

[4] R. Singh, L. Kishore, and N. Kaur, "Diabetic peripheral neuropathy: current perspective and future directions," Pharmacological Research, vol. 80, pp. 21-35, 2014.

[5] I. G. Obrosova, J. G. Mabley, Z. Zsengellér et al., "Role for nitrosative stress in diabetic neuropathy: evidence from studies with a peroxynitrite decomposition catalyst," FASEB Journal, vol. 19, no. 3, pp. 401-403, 2005.

[6] M.-C. Desco, M. Asensi, R. Márquez et al., "Xanthine oxidase is involved in free radical production in type 1 diabetes: protection by allopurinol," Diabetes, vol. 51, no. 4, pp. 1118-1124, 2002.

[7] U. R. Kuppusamy, M. Indran, and P. Rokiah, "Glycaemic control in relation to xanthine oxidase and antioxidant indices in Malaysian Type 2 diabetes patients," Diabetic Medicine, vol. 22, no. 10, pp. 1343-1346, 2005.

[8] D. J. Miric, B. B. Kisic, L. D. Zoric, R. V. Mitic, B. M. Miric, and I. M. Dragojevic, "Xanthine oxidase and lens oxidative stress markers in diabetic and senile cataract patients," Journal of Diabetes and Its Complications, vol. 27, no. 2, pp. 171-176, 2013.

[9] A. M. P. Feoli, F. E. Macagnan, C. H. Piovesan, L. C. Bodanese, and I. R. Siqueira, "Xanthine Oxidase activity is associated with risk factors for cardiovascular disease and inflammatory and oxidative status markers in metabolic syndrome: effects of a single exercise session," Oxidative Medicine and Cellular Longevity, vol. 2014, Article ID 587083, 8 pages, 2014.

[10] M. E. Inkster, M. A. Cotter, and N. E. Cameron, "Treatment with the xanthine oxidase inhibitor, allopurinol, improves nerve and vascular function in diabetic rats," European Journal of Pharmacology, vol. 561, no. 1-3, pp. 63-71, 2007.

[11] N. Papanas, N. Katsiki, K. Papatheodorou et al., "Peripheral neuropathy is associated with increased serum levels of uric acid in type 2 diabetes mellitus," Angiology, vol. 62, no. 4, pp. 291-295, 2011.

[12] D. Bar-Or, E. L. Lau, and J. V. Winkler, "A novel assay for cobaltalbumin binding and its potential as a marker for myocardial ischemia-a preliminary report," Journal of Emergency Medicine, vol. 19, no. 4, pp. 311-315, 2000.

[13] K. Ukinc, S. Eminagaoglu, H. O. Ersoz et al., "A novel indicator of widespread endothelial damage and ischemia in diabetic patients: ischemia-modified albumin," Endocrine, vol. 36, no. 3, pp. 425-432, 2009.

[14] S.-G. Ma, C.-L. Wei, B. Hong, and W.-N. Yu, "Ischemiamodified albumin in type 2 diabetic patients with and without peripheral arterial disease," Clinics, vol. 66, no. 10, pp. 16771680, 2011.

[15] J. W. G. Meijer, A. J. Smit, E. V. Sonderen, J. W. Groothoff, W. H. Eisma, and T. P. Links, "Symptom scoring systems to diagnose distal polyneuropathy in diabetes: the diabetic neuropathy symptom score," Diabetic Medicine, vol. 19, no. 11, pp. 962-965, 2002.

[16] E. L. Feldman, M. J. Stevens, P. K. Thomas, M. B. Brown, N. Canal, and D. A. Greene, "A practical two-step quantitative clinical and electrophysiological assessment for the diagnosis and staging of diabetic neuropathy," Diabetes Care, vol. 17, no. 11, pp. 1281-1289, 1994.

[17] K. V. Lemley, I. Abdullah, B. D. Myers et al., "Evolution of incipient nephropathy in type 2 diabetes mellitus," Kidney International, vol. 58, pp. 1228-1237, 2000.

[18] G. G. Roussos, "Xanthine oxidase from bovine small intestine," Methods in Enzymology, vol. 12, pp. 5-16, 1967.

[19] E. Beutler, O. Duron, and B. M. Kelly, "Improved method for the determination of blood glutathione," The Journal of Laboratory and Clinical Medicine, vol. 61, pp. 882-888, 1963.

[20] M. Kalousová, J. Škrha, and T. Zima, "Advanced glycation endproducts and advanced oxidation protein products in patients with diabetes mellitus," Physiological Research, vol. 51, no. 6, pp. 597-604, 2002. 
[21] B. Anderstam, B.-H. Ann-Christin, A. Valli, P. Stenvinkel, B. Lindholm, and M. E. Suliman, "Modification of oxidative stress biomarker AOPP assay: application in uremic samples," Clinica Chimica Acta, vol. 393, no. 2, pp. 114-118, 2008.

[22] M. Dobiášová and J. Frohlich, “The plasma parameter log (TG/HDL-C) as an atherogenic index: correlation with lipoprotein particle size and esterification rate inapob-lipoproteindepleted plasma $\left(\mathrm{FER}_{\mathrm{HDL}}\right)$

," Clinical Biochemistry, vol. 34, no. 7, pp. 583-588, 2001.

[23] A. M. Vincent, J. W. Russell, P. Low, and E. L. Feldman, "Oxidative stress in the pathogenesis of diabetic neuropathy," Endocrine Reviews, vol. 25, no. 4, pp. 612-628, 2004.

[24] A. C. P. Oliveira, C. J. Teixeira, T. F. Stefanello et al., "Oxidative stress parameters as biomarkers of risk factor for diabetic foot among the patients with type 2 diabetes," Brazilian Archives of Biology and Technology, vol. 57, no. 2, pp. 223-227, 2014.

[25] A. Altuntas, S. S. Goksu, V. Kidir, Z. D. Aydin, and M. T. Sezer, "Uric acid levels are inversely correlated with endothelial function in type 2 diabetic patients," International Journal of Clinical and Experimental Medicine, vol. 9, pp. 14105-14113, 2016.

[26] R. Wada and S. Yagihashi, "Role of advanced glycation end products and their receptors in development of diabetic neuropathy," Annals of the New York Academy of Sciences, vol. 1043, pp. 598-604, 2005.

[27] D. Bansal, K. Gudala, H. Muthyala, H. P. Esam, R. Nayakallu, and A. Bhansali, "Prevalence and risk factors of development of peripheral diabetic neuropathy in type 2 diabetes mellitus in a tertiary care setting," Journal of Diabetes Investigation, vol. 5, no. 6, pp. 714-721, 2014.

[28] K. J. Cheung, I. Tzameli, P. Pissios et al., "Xanthine oxidoreductase is a regulator of adipogenesis and PPAR $\gamma$ activity," Cell Metabolism, vol. 5, no. 2, pp. 115-128, 2007.

[29] Y. Tsushima, H. Nishizawa, Y. Tochino et al., "Uric acid secretion from adipose tissue and its increase in obesity," Journal of Biological Chemistry, vol. 288, no. 38, pp. 27138-27149, 2013.

[30] G. H. Tesch, "Role of macrophages in complications of Type 2 diabetes," Clinical and Experimental Pharmacology and Physiology, vol. 34, no. 10, pp. 1016-1019, 2007.

[31] N.-H. Kim, S. Choi, E.-J. Han et al., "The xanthine oxidaseNFAT5 pathway regulates macrophage activation and TLRinduced inflammatory arthritis," European Journal of Immunology, vol. 44, no. 9, pp. 2721-2736, 2014.

[32] A. Ives, J. Nomura, F. Martinon et al., "Xanthine oxidoreductase regulates macrophage IL1 $\beta$ secretion upon NLRP3 inflammasome activation," Nature Communications, vol. 6, article 6555, 2015.

[33] J. A. Honorat, M. Kinoshita, T. Okuno et al., "Xanthine oxidase mediates axonal and myelin loss in a murine model of multiple sclerosis," PLoS ONE, vol. 8, no. 8, Article ID e71329, 2013.

[34] H. M. El-Bassossy and M. L. Watson, "Xanthine oxidase inhibition alleviates the cardiac complications of insulin resistance: effect on low grade inflammation and the angiotensin system," Journal of Translational Medicine, vol. 13, article 82, 2015.

[35] R. Butler, A. D. Morris, J. J. F. Belch, A. Hill, and A. D. Struthers, "Allopurinol normalizes endothelial dysfunction in type 2 diabetics with mild hypertension," Hypertension, vol. 35 , no. 3, pp. 746-751, 2000.

[36] J. George and A. D. Struthers, "Role of urate, xanthine oxidase and the effects of allopurinol in vascular oxidative stress," Vascular Health and Risk Management, vol. 5, pp. 265-272, 2009.
[37] M. Connor, "Allopurinol for pain relief: more than just crystal clearance?" British Journal of Pharmacology, vol. 156, no. 1, pp. 4-6, 2009.

[38] R. Pop-Busui, M. J. Stevens, D. M. Raffel et al., "Effects of triple antioxidant therapy on measures of cardiovascular autonomic neuropathy and on myocardial blood flow in type 1 diabetes: a randomised controlled trial," Diabetologia, vol. 56, no. 8, pp. 1835-1844, 2013. 


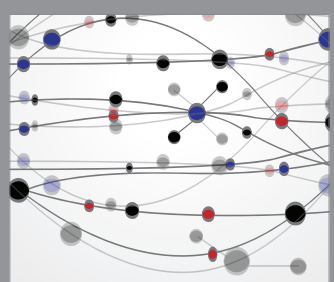

The Scientific World Journal
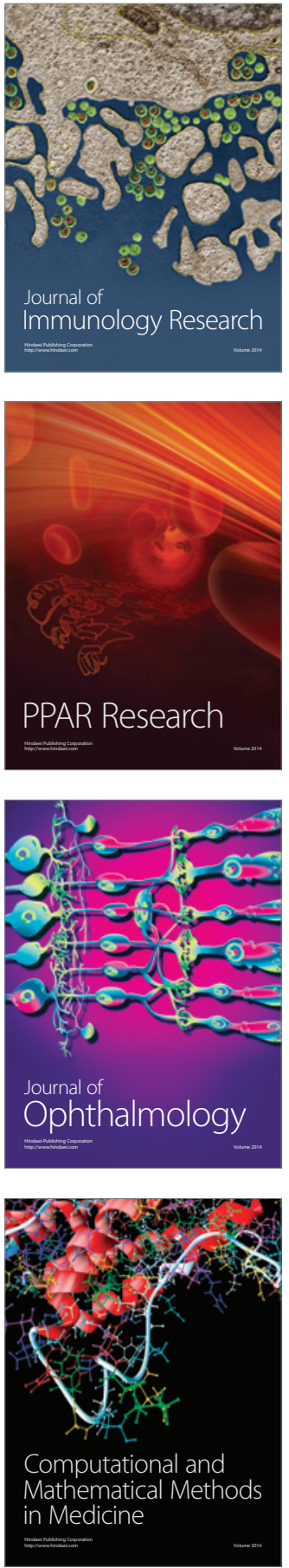

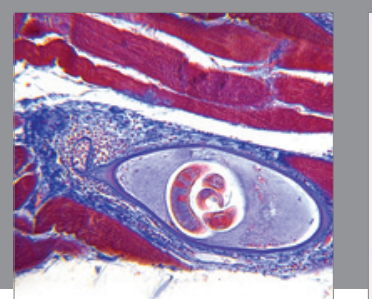

Gastroenterology Research and Practice

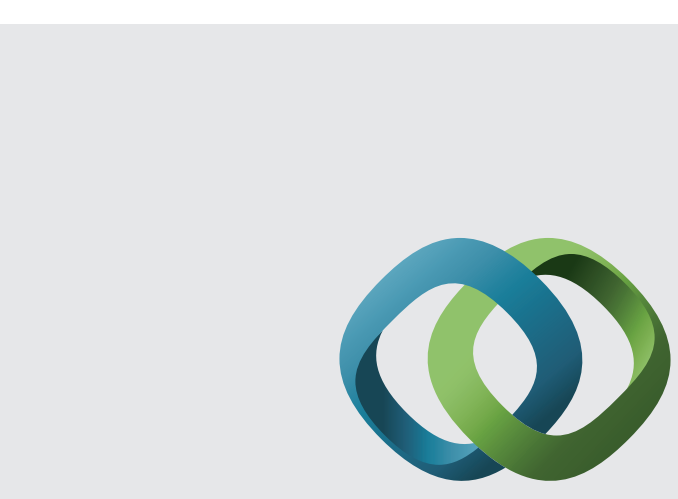

\section{Hindawi}

Submit your manuscripts at

http://www.hindawi.com
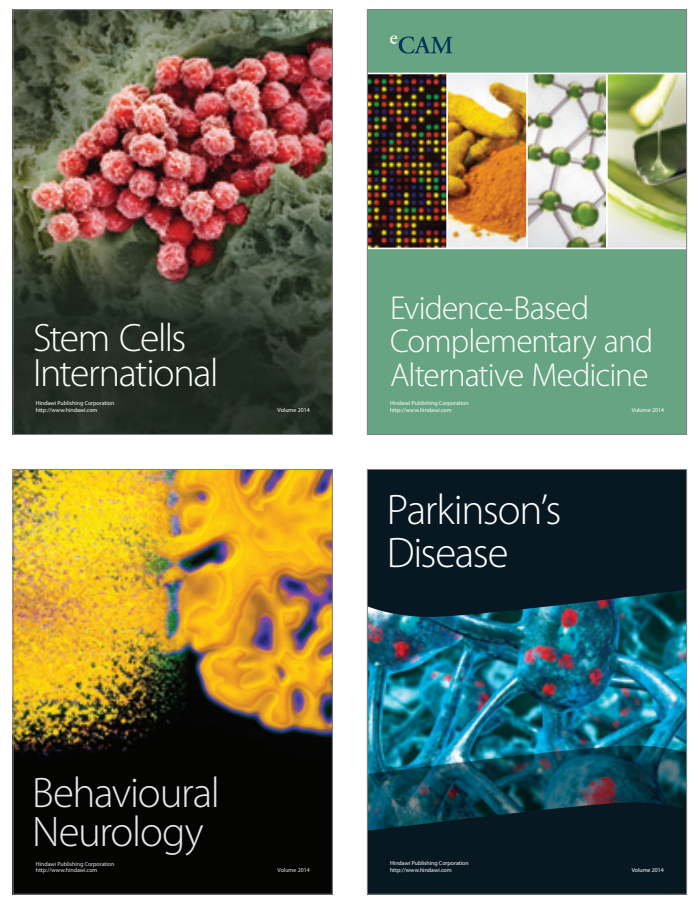
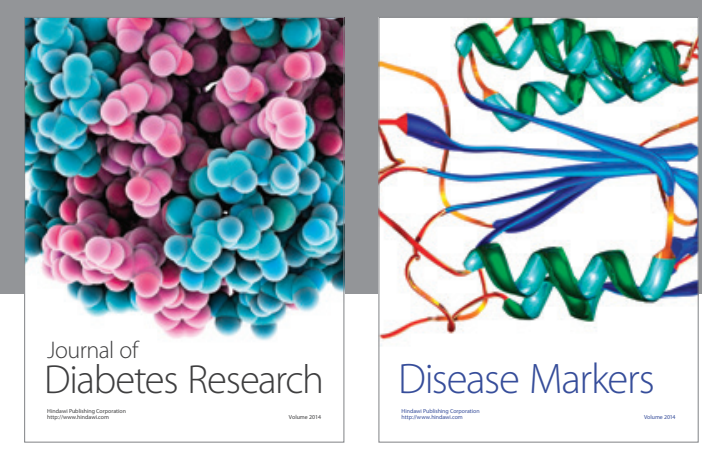

Disease Markers
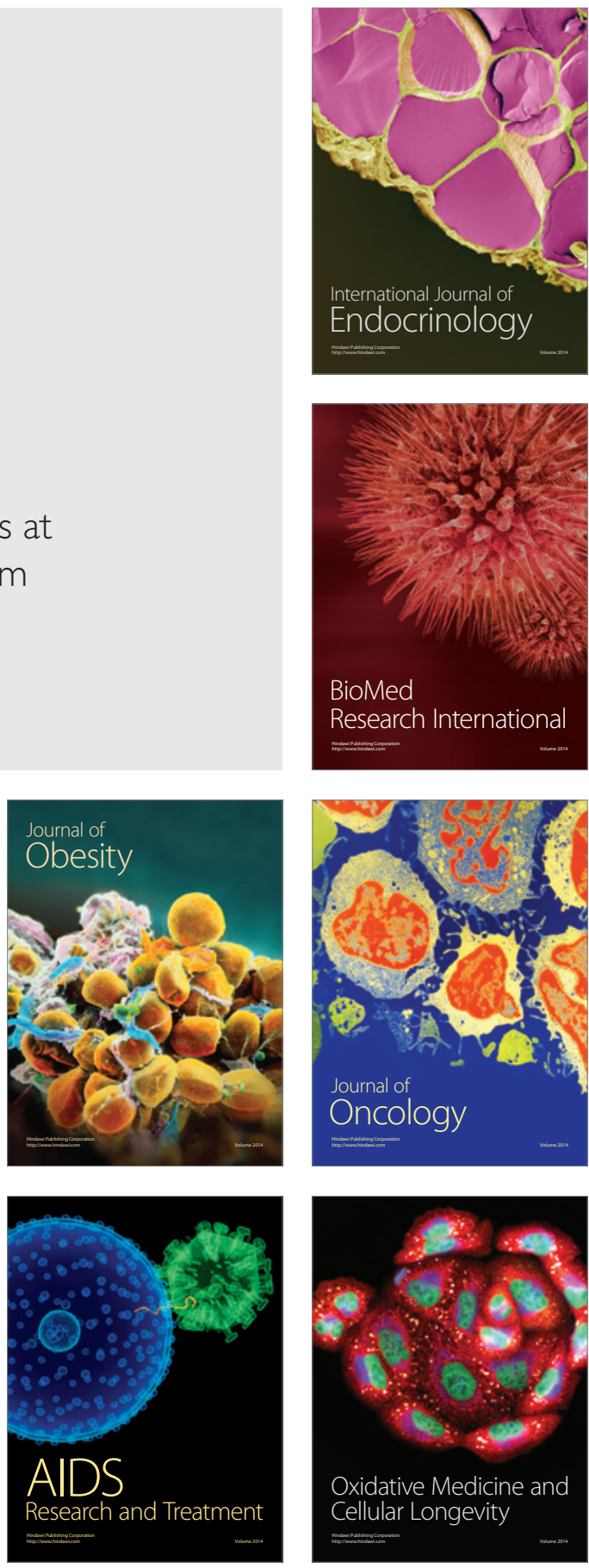